\title{
Gasping for a Diagnosis
}

\author{
Kristin D'Silva, M.D., \\ Department of Medicine, Brigham and Women's Hospital, Boston. \\ Sarah Brown, M.D., \\ Department of Medicine, Brigham and Women's Hospital, Boston. \\ Gary M. Hunninghake, M.D., \\ Department of Medicine, Brigham and Women's Hospital, Boston. \\ Marina Vivero, M.D., \\ Department of Pathology, Brigham and Women's Hospital, Boston. \\ Joseph Loscalzo, M.D., Ph.D. \\ Department of Medicine, Brigham and Women's Hospital, Boston.
}

\begin{abstract}
A 42-year-old man presented with progressive nonproductive cough and shortness of breath. At the onset of symptoms 6 months earlier, he had received a diagnosis of pneumonia and was treated with azithromycin. Two months before the current presentation, he was hospitalized at another institution for hypoxemia, and a computed tomographic (CT) scan of the chest showed ground-glass opacities in both lungs. Bronchoscopy with bronchoalveolar lavage was performed; the cytologic findings were reportedly normal, and Gram's staining and bacterial culture were negative. He was treated empirically with vancomycin, ceftriaxone, and prednisone and subsequently had modest improvement. He was discharged home with prednisone, at a dose of $60 \mathrm{mg}$ per day, and with supplemental oxygen that was to be administered through a nasal cannula at a rate of 2 liters per minute. In the subsequent weeks, he continued to have progressive symptoms and increased the supplemental oxygen to as much as 6 liters per minute through a nasal cannula. He reported no fevers, chills, night sweats, hemoptysis, or wheezing. He had lost $11 \mathrm{~kg}(25 \mathrm{lb})$ unintentionally over a 3-month period.
\end{abstract}

The patient presents with progressive cough, shortness of breath, hypoxemia, and groundglass opacities on imaging. These findings can be seen in patients with heart failure, various forms of interstitial lung disease, or cancer. His presentation is also notable for substantial unintentional weight loss, which arouses concern for cancer, chronic infections such as tuberculosis, and secondary infections due to human immunodeficiency virus (HIV). His symptoms have not abated despite treatment with two courses of antibiotic agents, which makes a typical bacterial infection a less likely diagnosis, although atypical processes,

\footnotetext{
Address reprint requests to Dr. Loscalzo at the Department of Medicine, Brigham and Women's Hospital, 75 Francis St., Boston, MA 02115, or at jloscalzo@ rics.bwh.harvard.edu.

Dr. Hunninghake reports receiving consulting fees from Genentech, Boehringer Ingelheim, Gerson Lehrman Group, and Mitsubishi Chemical. No other potential conflict of interest relevant to this article was reported.

Disclosure forms provided by the authors are available with the full text of this article at NEJM.org.
} 
including fungal and mycobacterial infections, remain possible. Among the various interstitial lung diseases, cryptogenic organizing pneumonia, hypersensitivity pneumonitis, and eosinophilic pneumonia are less likely diagnoses, given the fact that symptoms did not abate with glucocorticoid treatment. Interstitial lung diseases related to smoking, occupational exposure, or connective-tissue disorders (including myositis, rheumatoid arthritis, and systemic sclerosis) have variable response to glucocorticoids.

The patient had a history of hypertension and gastroesophageal reflux disease. He had been receiving lisinopril, omeprazole, prednisone, and trimethoprim-sulfamethoxazole, to which he reported full adherence. His family history was notable for rheumatoid arthritis in one cousin. He had lived in New Hampshire his entire life. He reported no recent travel or known contact with sick persons. He worked as a drywall installer. He had two pet dogs but no other animals. He had smoked one pack of cigarettes per day for 25 years but had quit 2 months before presentation to this hospital.

His smoking history is diagnostically pivotal and arouses concern for cancer, for smokingrelated interstitial lung diseases, such as respiratory bronchiolitis-associated interstitial lung disease or desquamative interstitial pneumonia, and for pulmonary alveolar proteinosis, all of which can manifest with progressive hypoxemia and ground-glass infiltrates. Interstitial lung disease that is associated with connective-tissue disorders, such as mixed connectivetissue disease, the antisynthetase syndrome, or vasculitis, is a concern, particularly given his family history of rheumatoid arthritis, and can manifest initially with pulmonary findings in the absence of other manifestations. His work as a drywall installer places him at risk for pneumoconiosis, given potential occupational exposures to asbestos, silica, talc, aluminum, and organic dusts, and for chronic hypersensitivity pneumonitis, given potential exposure to mold. Treatment with lisinopril can cause a dry cough but would not explain pulmonary infiltrates and hypoxemia. Although he is at risk for Pneumocystis jirovecii pneumonia because of his prolonged use of glucocorticoids, this infection would be unlikely, given that his symptoms preceded the initiation of treatment with glucocorticoids, and he has been adherent to prophylactic treatment with trimethoprim-sulfamethoxazole.

On physical examination, the patient's temperature was $37.2^{\circ} \mathrm{C}$, pulse 124 beats per minute, blood pressure $125 / 80 \mathrm{~mm} \mathrm{Hg}$, respiratory rate 30 breaths per minute, and oxygen saturation $81 \%$ while he was breathing oxygen through a nasal cannula at a rate of 6 liters per minute.

He appeared cachectic, with bitemporal wasting. His respirations were labored, and he used accessory muscles to breathe. The nasopharynx and oropharynx had no ulcerations. The jugular venous pressure was estimated to be $8 \mathrm{~cm}$ of water. Cardiac examination revealed tachycardia and a regular rhythm with no appreciable murmurs. The lungs had fine mid-tolate inspiratory crackles throughout both lung fields that were most prominent at the bases. No wheezing or rhonchi were noted. There was no clubbing, cyanosis, or edema in the arms or legs. The abdominal, neurologic, musculoskeletal, and cutaneous examinations were normal. High-flow oxygen therapy through a nasal cannula and empirical treatment with vancomycin, cefepime, and levofloxacin were initiated, and the patient was admitted to the intensive care unit. 
The patient is in acute respiratory distress. He is afebrile but is receiving prednisone, which may mask fevers and increase susceptibility to both typical and atypical pathogens. He appears euvolemic on examination, which lowers suspicion of heart failure. The fine crackles at mid-to-late inspiration suggest an interstitial process rather than a process of alveolar filling.

Standard chemical laboratory results and the white-cell count, differential, and platelet count were all within normal limits. The hemoglobin level was $19.1 \mathrm{~g}$ per deciliter, and the hematocrit was 56.6\%. Arterial blood gas analysis of a sample obtained while the patient was receiving high-flow oxygen therapy through a nasal cannula at a rate of 35 liters per minute, which probably resulted in a fraction of inspired oxygen $\left(\mathrm{FIO}_{2}\right)$ of near 1.0, showed a $\mathrm{pH}$ of 7.44, a partial pressure of carbon dioxide of $39 \mathrm{~mm} \mathrm{Hg}$, and a partial pressure of oxygen of $55 \mathrm{~mm} \mathrm{Hg}$. The calculated alveolar-arterial oxygen gradient, based on an $\mathrm{FIO}_{2}$ of 1.0, was $609 \mathrm{~mm} \mathrm{Hg}$ (by comparison, the expected alveolar-arterial gradient, based on the patient's age, would be $14.5 \mathrm{~mm} \mathrm{Hg}$ ).

The markedly elevated alveolar-arterial gradient in conjunction with the finding of diffuse ground-glass infiltrates arouses concern for interstitial lung disease - probably caused by a ventilation-perfusion mismatch, a limitation of diffusion, or both. The finding of marked polycythemia is probably the result of an appropriate rise in erythropoietin in response to sustained hypoxemia rather than caused by an erythropoietin-secreting tumor or polycythemia vera. Laboratory testing should be performed to evaluate the possibility of infectious and connective-tissue diseases, and a CT scan of the chest should be obtained.

The high-sensitivity C-reactive protein level was $36.6 \mathrm{mg}$ per liter (reference range, 0 to 3.0), and the erythrocyte sedimentation rate was $2 \mathrm{~mm}$ per hour (reference range, 0 to 12). The creatine kinase level was $76 \mathrm{U}$ per liter (reference range, 39 to 308), and the aldolase level was $6.7 \mathrm{U}$ per liter (normal value, <7.7). The lactate dehydrogenase level was $685 \mathrm{U}$ per liter (reference range, 135 to 225). The rheumatoid factor was $73 \mathrm{U}$ per milliliter (reference range, 0 to 15). Tests for antinuclear antibodies, antineutrophil cytoplasmic antibodies, anti-cyclic citrullinated peptide antibody, and anti-Jo-1 antibody were negative. Bacterial cultures from blood and sputum showed no growth. A respiratory viral panel and tests for serum fungal markers, HIV antibody, and urinary antigens for Streptococcus pneumoniae and Legionella pneumophila serogroup 1 were all negative.

A high C-reactive protein level with a low erythrocyte sedimentation rate can occur in the presence of bacterial infections, thromboembolic disease, or disseminated intravascular coagulation or early in the course of an inflammatory process, but this finding is nonspecific. Normal muscle enzymes and negative anti-Jo-1 antibody do not rule out myositis-related lung disease, since pulmonary manifestations can precede myositis; other myositis-specific antibodies should be checked. The lactate dehydrogenase level is substantially elevated and is a nonspecific marker of high cell turnover (such as in hemolysis or cancer), tissue injury (such as in pulmonary infarction or interstitial lung disease), or infection (such as $P$. jirovecii pneumonia). The positive rheumatoid factor does not necessarily suggest rheumatoid arthritis, since positive rheumatoid factor is present in 5\% of healthy persons and in some persons with other disorders, such as hepatitis and cryoglobulinemia, and in pulmonary 
interstitial processes, such as sarcoidosis. The absence of joint manifestations and the negative test for anti-cyclic citrullinated peptide antibody do not support a diagnosis of rheumatoid arthritis. Given the continued uncertainty in diagnosis, additional imaging should be obtained and bronchoscopy with bronchoalveolar lavage should be performed, although the use of glucocorticoids can decrease the yield of samples obtained from the lavage.

A CT scan of the chest showed that both lungs had consolidations with air bronchograms predominantly in the lower lobes and ground-glass opacities with interlobular septal thickening in the upper lobes, in a pattern termed "crazy paving," on the basis of its resemblance to irregularly placed paving stones (Fig. 1). The patient's condition continued to worsen, and his trachea was intubated because of progressive hypoxemia.

Crazy paving is a nonspecific radiographic pattern with ground-glass opacities, which suggest alveolar filling, and interlobular septal thickening, which suggests an interstitial process. Although crazy paving is classically associated with pulmonary alveolar proteinosis, the pattern is nonspecific and can be present in diverse processes, such as bronchioalveolar carcinoma, primary pulmonary lymphoma, sarcoidosis, lipoid pneumonia, the acute respiratory distress syndrome (ARDS), pulmonary edema, diffuse alveolar hemorrhage, and infections, including $P$. jirovecii pneumonia. However, the imaging findings combined with the negative workup thus far arouse concern for pulmonary alveolar proteinosis. Bronchoscopy with bronchoalveolar lavage is the diagnostic test of choice for this condition. In addition, bronchoscopy could be helpful in making alternate diagnoses, such as infection, cancer, eosinophilic pneumonia, and diffuse alveolar hemorrhage. If the diagnosis remains uncertain, surgical lung biopsy could provide a more definitive answer.

The decision was made to pursue simultaneous bronchoscopy with bronchoalveolar lavage and wedge biopsy. Bronchoalveolar lavage yielded cloudy fluid with 600 red cells per cubic millimeter and 11,500 total nucleated cells per cubic millimeter, of which $76 \%$ were neutrophils, $19 \%$ macrophages, $3 \%$ monocytes, $1 \%$ lymphocytes, and $1 \%$ eosinophils. A wedge biopsy specimen showed intact parenchymal architecture with abundant and diffusely distributed intraalveolar proteinaceous material that was positive on periodic acid-Schiff staining (indicating the presence of protein and glycogen). Periodic acid-Schiff staining with diastase digestion, which eliminates glycogen, was also positive, a finding that confirmed the proteinaceous nature of the intraalveolar material (Fig. 2). There was no histologic evidence of inflammation, acute lung injury, airway disease, interstitial fibrosis, or pleural disease. The patient received a diagnosis of pulmonary alveolar proteinosis.

A bronchoalveolar lavage with cloudy fluid that is positive on periodic acid-Schiff staining is diagnostic of pulmonary alveolar proteinosis, and lung biopsy is generally not necessary. Treatment includes therapeutic whole-lung bronchoalveolar lavage and subcutaneous or inhaled granulocyte-macrophage colony-stimulating factor (GM-CSF).

The patient underwent three therapeutic whole-lung bronchoalveolar lavages, with the total volume of lavage fluid limited by hypoxemia. Treatment with subcutaneous GM-CSF at a dose of $250 \mu \mathrm{g}$ per day was initiated. At the most recent follow-up visit, 1 year after 
initiation of GM-CSF, the patient was not using supplemental oxygen and had returned to normal activities.

\section{Commentary}

The patient presented with recurrent and progressive episodes of hypoxemia over the course of 6 months, with imaging revealing diffuse ground-glass opacities with interlobular septal thickening in both lungs, in a pattern known as crazy paving. When his trachea was intubated because of progressive hypoxemia, bronchoscopy with bronchoalveolar lavage and wedge resection biopsy established the diagnosis of pulmonary alveolar proteinosis.

Pulmonary alveolar proteinosis is a rare disorder that is marked by alveolar filling with a lipid-rich proteinaceous material accompanied by large, foamy, alveolar macrophages and relatively few inflammatory cells. ${ }^{1}$ The median age at diagnosis is 39 years, and most patients are male, with approximately $70 \%$ having a history of smoking. ${ }^{1}$ Pulmonary alveolar proteinosis most commonly manifests with progressive exertional dyspnea, nonproductive cough, fatigue, and weight loss. Whereas the results of standard laboratory tests are often normal, the lactate dehydrogenase level is usually elevated and may be a useful marker of the severity of the disease. ${ }^{1}$ Although crazy paving observed on CT is classically associated with pulmonary alveolar proteinosis, in a case series involving 99 patients whose imaging showed crazy paving, only 1 patient had pulmonary alveolar proteinosis, and the most common causes of crazy paving were infection, ARDS, and pulmonary edema. ${ }^{2}$ Among patients with pulmonary alveolar proteinosis, bronchoalveolar lavage yields milky opaque fluid that is positive on periodic acid-Schiff staining. Although lung biopsy is generally not needed for diagnosis, it is notable for preserved lung architecture with alveolar filling by histologically distinctive coarse and densely eosinophilic material that is positive on periodic acid-Schiff staining. ${ }^{1}$ The natural history of the disease is variable; patients may have stable persistent symptoms, progressive deterioration, or spontaneous remission, with spontaneous remission occurring in 5 to $7 \%$ of patients. ${ }^{1,3}$ In a retrospective analysis of 343 cases, the percentage of patients who were alive at 5 years before the availability of current therapies — was $75 \%$; major causes of death included progressive respiratory failure and pulmonary infections. ${ }^{1}$

Pulmonary alveolar proteinosis can be hereditary, secondary, or autoimmune. The hereditary form, which accounts for less than $1 \%$ of cases, arises from mutations in surfactant protein $\mathrm{B}$ or $\mathrm{C}$, the receptor for GM-CSF, $A B C A 3$, thyroid transcription factor 1, or several other mutations. ${ }^{3,4}$ Secondary pulmonary alveolar proteinosis, which accounts for $9 \%$ of cases, develops under conditions that result in alveolar macrophage dysfunction or deficiency, such as inhalational exposure (e.g., to silica dust or toxic fumes), immunosuppression, infections, or cancer (most commonly myelodysplastic syndrome and acute myeloid leukemia). ${ }^{3}$ The autoimmune form is responsible for approximately $90 \%$ of cases and results from the development of antibodies that target GM-CSF. ${ }^{3}$ Clinical testing for serum GM-CSF autoantibodies is commercially available, and the combination of antibody testing and bronchoalveolar lavage obviates the need for lung biopsy in most patients. ${ }^{3}$ 
GM-CSF is a hematologic growth factor that has receptors on alveolar type II epithelial cells (which produce surfactant), monocytes, and macrophages. Surfactant is a complex mixture of lipids and proteins that reduces surface tension at the air-alveolar interface, thus preventing alveolar collapse, and plays a role in innate immunity. GM-CSF stimulates the maturation of alveolar macrophages, which are pivotal to surfactant catabolism. ${ }^{4}$ Knockout mice that are deficient in GM-CSF have alveoli that contain large macrophages that have the ability to ingest but not catabolize surfactant. ${ }^{5-7}$ Similarly, in humans with autoimmune pulmonary alveolar proteinosis, autoantibodies against GM-CSF lead to dysfunction in alveolar macrophages, which results in impaired catabolism and accumulation of surfactant lipids and proteins. ${ }^{8}$

The therapeutic approach to pulmonary alveolar proteinosis depends on the cause. For hereditary pulmonary alveolar proteinosis, treatment generally includes supportive care or lung transplantation; whole-lung lavage is usually ineffective, and new therapies such as macrophage transplantation are being investigated. ${ }^{9}$ For secondary pulmonary alveolar proteinosis, the treatment targets the underlying condition (e.g., hematopoietic stem-cell transplantation for hematologic cancer). Autoimmune pulmonary alveolar proteinosis is treated with whole-lung lavage, which involves general anesthesia, isolation of the lung with a double-lumen endotracheal tube and single-lung ventilation, and instillation of the other lung with 1-liter aliquots of normal saline followed by chest physiotherapy and drainage of the proteinaceous effluent with the help of postural positioning; an average total volume of 15 liters is used per lung, and the average number of lavages performed is 2.5 over a 5 -year period. ${ }^{4,10}$ In observational studies, whole-lung lavage has been associated with reduced mortality, a substantial reduction in symptoms, and improvement in pulmonary function tests. In a retrospective analysis of data from 146 patients, the mean percentage of patients who were alive at 5 years was $94 \%$ with lavage as compared with $85 \%$ without lavage $(\mathrm{P}=$ 0.04). ${ }^{10}$ Subcutaneous or inhaled GM-CSF therapy has been reported in uncontrolled clinical trials to result in improvement in alveolar-arterial oxygen gradient and in quality of life, but long-term effects, including effects on mortality, have not been studied, and data from controlled clinical trials are needed. ${ }^{11-14}$ Therapies considered for refractory disease, on the basis of case reports, include rituximab, therapeutic plasma exchange, and lung transplantation, although the disease can recur in the lung allograft. ${ }^{4}$

The current case highlights the importance of considering alternative diagnoses when patients who have progressive pulmonary infiltrates and hypoxemia do not show improvement with antibiotics. Pulmonary alveolar proteinosis should be considered as a potential diagnosis in patients with a history of smoking or other inhalational exposures in whom a nonproductive cough and progressive hypoxemia develop and crazy paving is seen on imaging. In the current case, identification of pulmonary alveolar proteinosis and subsequent appropriate treatment resulted in marked clinical improvement.

\section{Acknowledgments}

We thank Dr. Bruce Levy for his assistance with the preparation of an earlier version of the manuscript. 


\section{REFERENCES}

1. Trapnell BC, Whitsett JA, Nakata K. Pulmonary alveolar proteinosis. N Engl J Med 2003;349:252739. [PubMed: 14695413]

2. De Wever W, Meersschaert J, Coolen J, Verbeken E, Verschakelen JA. The crazy-paving pattern: a radiological-pathological correlation. Insights Imaging 2011;2:117-32. [PubMed: 22347941]

3. Suzuki T, Trapnell BC. Pulmonary alveolar proteinosis syndrome. Clin Chest Med 2016;37:431-40. [PubMed: 27514590]

4. Kumar A, Abdelmalak B, Inoue Y, Culver DA. Pulmonary alveolar proteinosis in adults: pathophysiology and clinical approach. Lancet Respir Med 2018;6:554-65. [PubMed: 29397349]

5. Stanley E, Lieschke GJ, Grail D, et al. Granulocyte/macrophage colony-stimulating factor-deficient mice show no major perturbation of hematopoiesis but develop a characteristic pulmonary pathology. Proc Natl Acad Sci U S A 1994;91:5592-6. [PubMed: 8202532]

6. Dranoff G, Crawford AD, Sadelain M, et al. Involvement of granulocyte-macrophage colonystimulating factor in pulmonary homeostasis. Science 1994;264:713-6. [PubMed: 8171324]

7. Ikegami M, Ueda T, Hull W, et al. Surfactant metabolism in transgenic mice after granulocyte macrophage-colony stimulating factor ablation. Am J Physiol 1996; 270:L650-L658. [PubMed: 8928826]

8. Kitamura T, Tanaka N, Watanabe J, et al. Idiopathic pulmonary alveolar proteinosis as an autoimmune disease with neutralizing antibody against granulocyte/macrophage colony-stimulating factor. J Exp Med 1999;190:875-80. [PubMed: 10499925]

9. Doerschuk CM. Pulmonary alveolar proteinosis and macrophage transplantation. N Engl J Med 2015;372:1762-4. [PubMed: 25923557]

10. Michaud G, Reddy C, Ernst A. Whole-lung lavage for pulmonary alveolar proteinosis. Chest 2009;136:1678-81. [PubMed: 19995769]

11. Seymour JF, Dunn AR, Vincent JM, Presneill JJ, Pain MC. Efficacy of granulocyte-macrophage colony-stimulating factor in acquired alveolar proteinosis. N Engl J Med 1996;335:1924-5. [PubMed: 8965913]

12. Seymour JF, Presneill JJ, Schoch OD, et al. Therapeutic efficacy of granulocyte-macrophage colony-stimulating factor in patients with idiopathic acquired alveolar proteinosis. Am J Respir Crit Care Med 2001;163:524-31. [PubMed: 11179134]

13. Venkateshiah SB, Yan TD, Bonfield TL, et al. An open-label trial of granulocyte macrophage colony stimulating factor therapy for moderate symptomatic pulmonary alveolar proteinosis. Chest 2006; 130:227-37. [PubMed: 16840407]

14. Tazawa R, Trapnell BC, Inoue Y, et al. Inhaled granulocyte/macrophage-colony stimulating factor as therapy for pulmonary alveolar proteinosis. Am J Respir Crit Care Med 2010;181:1345-54. [PubMed: 20167854] 

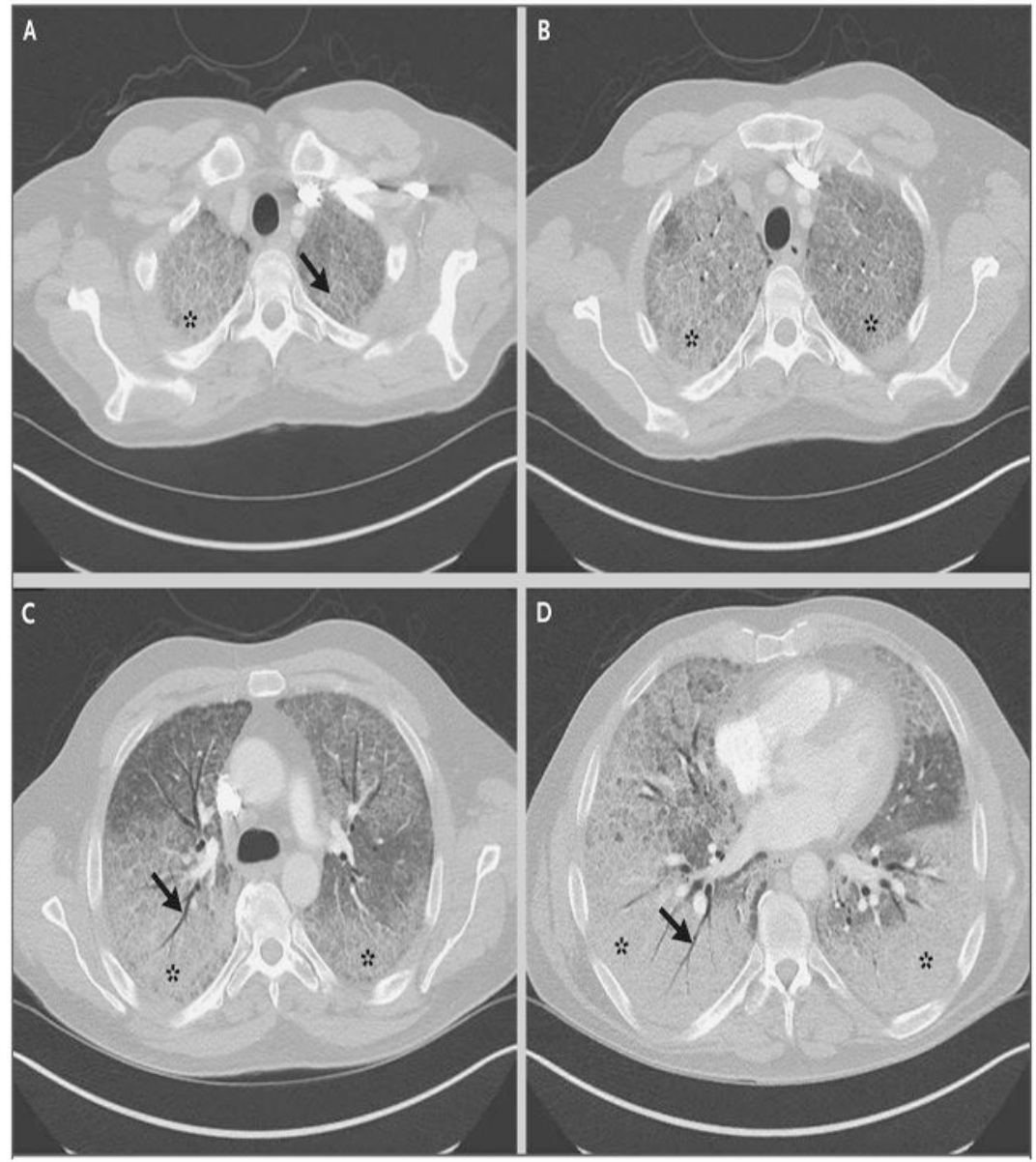

Figure 1. Computed Tomographic Images of the Chest.

Panels A and B show ground-glass opacities (asterisks) with interlobular septal thickening (Panel A, arrow) in the upper lobes of the lungs. Panels C and D show lower lobepredominant consolidations (asterisks) with air bronchograms (arrows) in both lungs. 

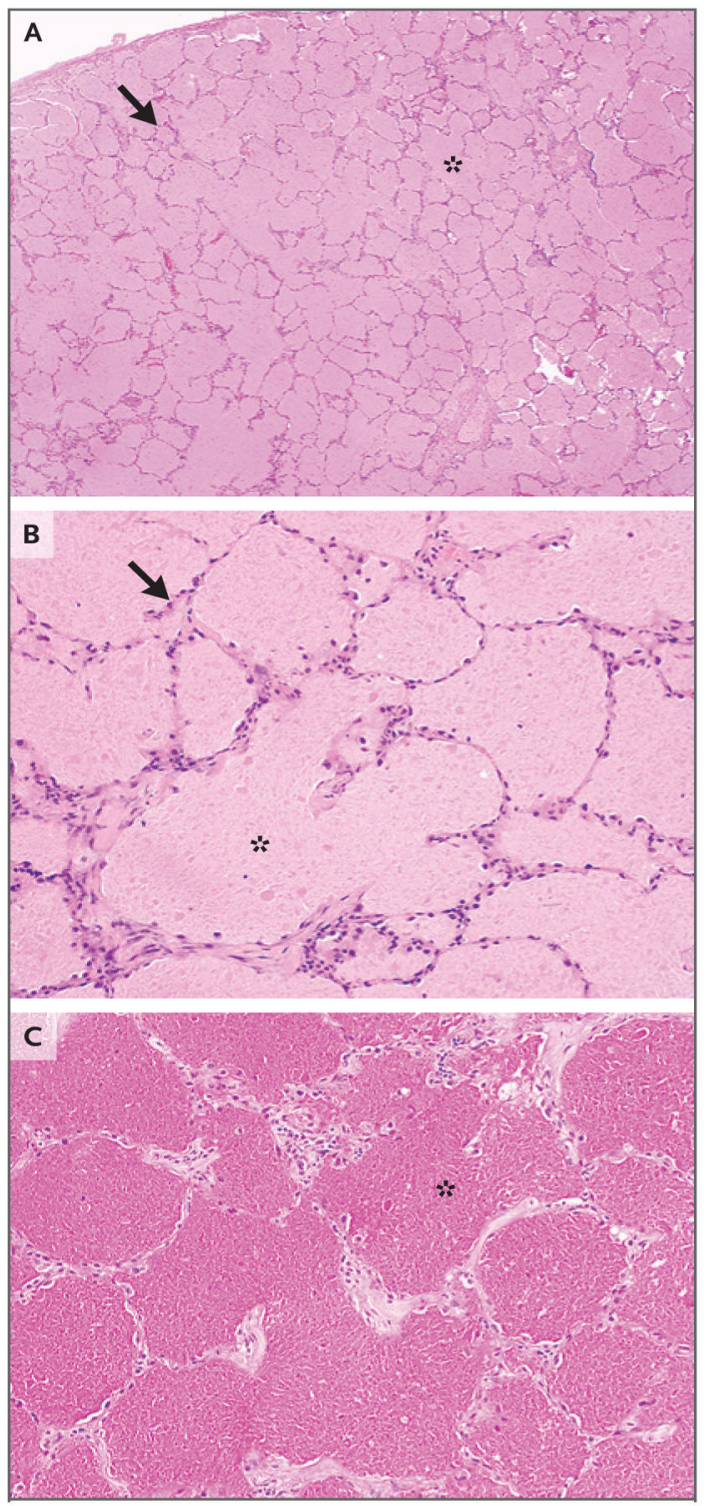

Figure 2. Biopsy Specimen of the Left Lung.

In Panel A, and in Panel B at higher magnification, hematoxylin and eosin staining of a biopsy specimen shows preserved lung architecture (arrows) with abundant coarse intraalveolar eosinophilic material (asterisks), findings that are characteristic of pulmonary alveolar proteinosis. As shown in Panel C, the result of periodic acid-Schiff staining with diastase digestion to eliminate glycogen was positive, which confirmed the proteinaceous nature of the intraalveolar material (asterisk). 